\title{
Uso da FISH em mucosa oral para investigação de mosaicismo com linhagem 45, $X$ : estudo com homens saudáveis e pacientes com distúrbios da diferenciação do sexo
}

\author{
The use of FISH on buccal smear to investigate \\ mosaicism with a 45, $X$ cell line: study on healthy men \\ and patients with disorders of sex development
}

'Programa de Pós-Graduação em Ciências Médicas, Faculdade de Ciências Médicas, Universidade Estadual de Campinas (FCM) Unicamp), Campinas, SP, Brasil 2 Programa de Pós-Graduação em Saúde da Criança e do Adolescente, FCM/Unicamp, Campinas, SP, Brasil ${ }^{3}$ Departamento de Genética Médica, FCM/Unicamp Campinas, SP, Brasil

${ }^{4}$ Departamento de Pediatria, FCM/ Unicamp, Campinas, SP, Brasil

Trabalho realizado no Departamento de Genética Médica, Faculdade de Ciências Médicas, Universidade Estadual de Campinas

Juliana de Paulo', Juliana Gabriel Ribeiro de Andrade' ${ }^{2}$ Ana Paula dos Santos', Vera Lúcia Gil-da-Silva-Lopess ${ }^{3}$, Gil Guerra-Júnior ${ }^{4}$, Andréa Trevas Maciel-Guerra ${ }^{3}$

\section{RESUMO}

Objetivo: Verificar se a hibridização in situ por fluorescência (FISH) em células de mucosa oral poderia ser empregada para detectar criptomosaicismo com linhagem $45, X$ em pacientes 46,XY. Sujeitos e métodos: Amostra de 19 jovens saudáveis $46, X Y$ e cinco pacientes com distúrbios da diferenciação do sexo (DDS), quatro 45,X/46,XY e um 46,XY. FISH com sondas específicas para $\mathrm{X}$ e $\mathrm{Y}$ em núcleos interfásicos de linfócitos e mucosa oral para investigar a proporção de núcleos contendo apenas o sinal do cromossomo $X$. Resultados: A frequência de núcleos contendo apenas o sinal do $X$ nos dois tecidos dos homens saudáveis não diferiu $(p=$ 0,69 ). Em todos os pacientes com DDS essa frequência foi significativamente maior, e também não houve diferença entre os dois tecidos $(p=0,38)$. Conclusões: A investigação de mosaicismo com linhagem $45, X$ em pacientes com DDS $46, \mathrm{XY}$ ou esterilidade pode ser feita por FISH diretamente em células de mucosa oral. Arq Bras Endocrinol Metab. 2014;58(4):328-34

Descritores

Mosaicismo; cromossomos sexuais; hibridização in situ por fluorescência; transtornos do desenvolvimento sexual

Correspondência para: Andréa Trevas Maciel Guerra Departamento de Genética Médica, Faculdade de Ciências Médicas, Universidade Estadual de Campinas Rua Tessália Vieira de Camargo, 126 13083-887 - Campinas, SP, Brasil atmg@uol.com.br

Recebido em 24/Jun/2013 Aceito em 25/Nov/2013

DOI: 10.1590/0004-2730000002853

\section{ABSTRACT}

Objective: To verify whether fluorescence in situ hybridization (FISH) of cells from the buccal epithelium could be employed to detect cryptomosaicism with a $45, X$ lineage in $46, X Y$ patients. Subjects and methods: Samples of nineteen $46, X Y$ healthy young men and five patients with disorders of sex development (DSD), four $45, X / 46, X Y$ and one $46, X Y$ were used. FISH analysis with $X$ and $Y$ specific probes on interphase nuclei from blood lymphocytes and buccal epithelium were analyzed to investigate the proportion of nuclei containing only the signal of the $X$ chromosome. Results: The frequency of nuclei containing only the $X$ signal in the two tissues of healthy men did not differ $(p=0.69)$. In all patients with DSD this frequency was significantly higher, and there was no difference between the two tissues $(p=0.38)$, either. Conclusions: Investigation of mosaicism with a $45, X$ cell line in patients with $46, X Y$ DSD or sterility can be done by FISH directly using cells from the buccal epithelium. Arq Bras Endocrinol Metab. 2014;58(4):328-34

\section{Keywords}

Mosaicism; sex chromosomes; in situ hybridization, fluorescence; disorders of sex development 


\section{INTRODUÇÃO}

A $\mathrm{s}$ anomalias cromossômicas numéricas e estruturais são importante causa de morbidade e mortalidade e podem envolver autossomos e( $\mathrm{ou}$ ) cromossomos sexuais. As anomalias numéricas se devem a problemas durante a divisão de células germinativas ou somáticas por falta de disjunção ou perda cromossômica, esta última por atraso anafásico ou pelo fato de o cromossomo ser estruturalmente anômalo. Quando esses eventos ocorrem na mitose podem ocasionar mosaicismo, ou seja, presença de duas ou mais linhagens cromossomicamente distintas (1). Estudos no período inicial do desenvolvimento embrionário após fertilização in vitro sugerem que o mosaicismo ocorre em $50 \%$ dos embriões até o estágio de oito células e $75 \%$ dos blastocistos (2).

Quanto mais precoce a ocorrência do mosaicismo (na primeira divisão mitótica do zigoto ou durante a divisão de um dos blastômeros, por exemplo), maior a proporção de células anômalas e, portanto, mais evidentes as consequências clínicas. Se apenas uma pequena fração das células for anômala, o mosaicismo pode nunca ser detectado (1).

As anomalias dos cromossomos sexuais incidem em cerca de 1:500 nascimentos e se associam com frequência a mosaicismo. Os fenótipos associados a elas são, em geral, menos exuberantes do que os das anomalias autossômicas, já que a inativação do X e o pequeno número de genes do Y reduzem as consequências clínicas do desequilíbrio do material cromossômico (3). Algumas dessas anomalias determinam esterilidade (síndromes de Turner (ST) e de Klinefelter) ou baixa estatura (ST) e outras são associadas à estatura mais elevada, porém geralmente com desenvolvimento puberal e fertilidade normais $(47, \mathrm{XYY}$ e $47, \mathrm{XXX})$.

$\mathrm{Na}$ ST, além do cariótipo $45, \mathrm{X}$ são encontrados diversos tipos de mosaicos sem alterações estruturais $(45, \mathrm{X} / 46, \mathrm{XX} ; \quad 45, \mathrm{X} / 47, \mathrm{XXX} ; 45, \mathrm{X} / 46, \mathrm{XY}$; $45, \mathrm{X} / 47, \mathrm{XYY})$ e diversas alterações estruturais do cromossomo X ou Y com ou sem mosaicismo com linhagem 45,X (como isocromossomos, deleções e cromossomos em anel). Em menos de 10\% dos casos, há cromossomo Y na constituição cromossômica, seja ele íntegro ou com alteração estrutural (4).

A baixa estatura e o hipogonadismo hipergonadotrófico são os sinais mais constantes da ST, seguidos de diversos dismorfismos, anomalias cardiovasculares, renais e renovasculares e afecções adquiridas, como doença tiroidiana auto-imune, entre outros achados (5).
Essas mesmas características podem ser observadas em pacientes com cariótipo 45,X/46, XY e outros fenótipos gonadais e genitais: a disgenesia gonadal mista (DGM), na qual há ambiguidade genital e gônada em fita associada a testículo disgenético ou dois testículos disgenéticos; o hermafroditismo verdadeiro ou distúrbio da diferenciação do sexo ovário-testicular, caracterizado por ambiguidade genital e coexistência de tecido ovariano e testicular; e quadros de esterilidade em homens com genitália masculina $(6,7)$. Essa variabilidade fenotípica está associada às diferentes proporções das linhagens 45,X e 46,XY nas gônadas (8).

A perda do cromossomo $\mathrm{Y}$ gerando células $45, \mathrm{X}$ pode ser observada também em indivíduos do sexo masculino que não apresentam anomalias do aparelho reprodutor. Há correlação altamente significativa entre a perda desse cromossomo e a idade em homens saudáveis, sendo esta mais acentuada a partir dos 16 anos (9). Além disso, a perda desse cromossomo em grande número de células também pode ser observada em tecidos neoplásicos (10).

A DGM tem como diagnóstico diferencial a disgenesia gonadal parcial 46,XY (DGP XY), caracterizada por ambiguidade genital com cariótipo $46, \mathrm{XY}$ sem mosaicismo em pacientes com diferenciação testicular parcial, presença de derivados dos dutos de Müller e sem sinais clínicos da ST. Na maioria dos casos a etiologia é desconhecida, mas já foram descritas mutações nos genes SRY e NR5AI (11,12). A distinção entre DGP $\mathrm{XY}$ e DGM se dá por meio do exame de cariótipo, buscando afastar com alto grau de probabilidade a presença de linhagem $45, X$.

A partir do final dos anos 1980, a hibridação in situ por fluorescência (fluorescence in situ hybridisation, FISH) passou a ser incorporada à rotina de análise cromossômica. Essa técnica consiste no uso de sondas de DNA marcado por fluorescência para identificar todo um cromossomo ("pintura cromossômica"), um local específico no cromossomo, ou apenas sua região centromérica (13). Em casos de ST, tem sido aplicada na identificação da origem de cromossomos estruturalmente anômalos e também na pesquisa de mosaicismo, pois permite a análise rápida de centenas de células, tanto metáfases quanto núcleos interfásicos de diversos tecidos (13).

Assim, o uso de FISH com sondas centroméricas de $\mathrm{X}$ e $\mathrm{Y}$ permite investigar se cromossomos marcadores são derivados do $\mathrm{Y}$, identificando os casos em que há risco de tumores gonadais (14). Permite ainda investigar a presença de outras linhagens quando há somente 
células 45,X no cariótipo convencional e também identificar uma linhagem 45,X nos casos em que, apesar de forte suspeita clínica, o cariótipo encontra somente células 46,XX (4).

Devido ao risco de neoplasias gonadais, vários pesquisadores têm buscado o diagnóstico de mosaicismo com linhagem contendo cromossomo $\mathrm{Y}$ em pacientes com ST e cariótipo 45,X aparentemente homogêneo (14). No entanto, é pouco estudada a questão inversa, ou seja, as estratégias de detecção de linhagem $45, \mathrm{X}$ em pacientes com ambiguidade genital ou esterilidade e cariótipo 46,XY aparentemente homogêneo. Neles, a falta de detecção do mosaicismo faz com que deixem de ser investigadas precocemente diversas afecções congềnitas e adquiridas.

O mosaicismo críptico pode não ser detectado pelo estudo de pequeno número de metáfases de linfócitos (de origem mesodérmica), como ocorre no exame de cariótipo rotineiro, exigindo ampliação do número de células analisadas, seja no cariótipo convencional ou por meio de FISH, ou mesmo o estudo de outros tecidos $(15,16)$. O cariótipo a partir de culturas de fibroblastos (de origem ectodérmica) pode ser empregado na pesquisa de mosaicismo, porém é feito por meio de procedimento mais invasivo (biópsia de pele), que exige maior tempo de cultura e maior gasto de material de consumo e de recursos humanos, trazendo, portanto, maior custo, além de ser mais suscetível à contaminação. Nesse tipo de cultura, há ainda resistência frequente das células à ação da colchicina e possibilidade de ocorrência de pseudomosaicismo in vitro. Esta, portanto, não é a técnica ideal para ser empregada como rotina no diagnóstico.

Um método não invasivo, mais rápido e simples que a cultura de fibroblastos, como a análise de células obtidas de raspado do epitélio bucal (também de origem ectodérmica) por meio da aplicação da técnica de FISH, seria de grande auxílio na detecção rotineira de mosaicismo em baixa frequência nesses pacientes. Assim, o objetivo deste trabalho foi verificar se a técnica de FISH em células de mucosa oral poderia ser empregada na investigação de mosaicismo críptico com linhagem $45, \mathrm{X}$ em pacientes com DDS ou esterilidade e cariótipo 46,XY.

\section{SUJEITOS E MÉTODOS}

A casuística foi composta por 19 homens saudáveis com idades entre 21 e 29 anos (média: 23 anos) com carió- tipo 46,XY em 50 células analisadas, o que permitiu afastar a existência de mosaicismo de $6 \%$ ou mais com 95\% de confiabilidade (15). Foram critérios de exclusão antecedentes pessoais de genitoplastias (orquidopexia, uretroplastia) e terapia de reposição com hormônios masculinos para indução e/ou manutenção de caracteres sexuais secundários masculinos, presença de anomalias congênitas cardíacas, renais e de vias urinárias, doença tiroidiana autoimune e baixa estatura.

Um segundo grupo analisado foi constituído por cinco pacientes com distúrbios da diferenciação do sexo (DDS) cujas idades variaram de 5 a 23 anos (média: 12,6 anos), três com sexo social masculino e dois, feminino. Um dos pacientes possuía diagnóstico prévio de DGP 46,XY e vinha apresentando déficit de crescimento durante o seguimento, o que gerou suspeita de mosaicismo críptico com linhagem $45, \mathrm{X}$; os demais tinham cariótipo $45, \mathrm{X} / 46, \mathrm{XY}$, dos quais três com DGM e um com ST (Tabela 1).

Tabela 1. Diagnóstico clínico e constituição cromossômica dos cinco pacientes com distúrbios da diferenciação do sexo que fizeram parte da casuística deste trabalho

\begin{tabular}{lcc}
\hline Caso & Diagnóstico clínico & Cariótipo \\
\hline 1A & DGM & $45, X[2] / 46, X Y[48]$ \\
$2 A$ & $D G P ~ X Y$ & $46, X Y[50]$ \\
$3 A$ & DGM & $45, X[34] / 46, X Y[16]$ \\
$4 A$ & ST & $45, X[14] / 46, X Y[36]$ \\
$5 A$ & DGM & $45, X / 46, X Y, \operatorname{del}(Y)(q 12)$ \\
\hline
\end{tabular}

DGM: disgenesia gonadal mista; DGP XY: disgenesia gonadal parcial 46,XY; ST: síndrome de Turner.

Nos indivíduos dos dois grupos foram analisados núcleos interfásicos de linfócitos de sangue periférico e mucosa oral por meio de FISH com sondas específicas para os cromossomos X e Y. Para a técnica de FISH foram utilizadas sondas tipo alfa-satélite lócus específica para as regiões centroméricas dos cromossomos X (DXZ1, marcada em verde) e Y (DYZ3, marcada em vermelho) (Cytocell Vysis, Inc., Downers Grove, IL). Nas preparações de linfócitos de sangue periférico foram seguidas as instruções do fabricante, enquanto para a realização da FISH em mucosa oral foi utilizado o protocolo adaptado proposto por Romczak e Punnett (17).

As células da mucosa bucal foram coletadas com uma haste de madeira das laterais internas da bochecha. A amostra foi colocada imediatamente em tubo contendo solução salina estéril, o qual foi centrifugado três vezes com salina e mais três vezes com solução fixadora (3:1 de metanol e ácido acético). Após 24 horas, as 
amostras foram pingadas em lâminas e armazenadas em estufa $65^{\circ} \mathrm{C}$ por 2 horas. As lâminas foram previamente avaliadas em microscópio de fase para seleção da área de hibridação. Após essa etapa, foram colocadas em solução de $2 \mathrm{xSSC}$ a $73^{\circ} \mathrm{C}$ por 2 minutos e colocadas em solução contendo pepsina $\left(\right.$ SIGMA $\left.^{\circledR}\right)$ 0,005\% em $10 \mathrm{mM} \mathrm{HCl}$ a $37^{\circ} \mathrm{C}$ por 40 minutos. As lâminas foram lavadas duas vezes no tampão PBS $1 \mathrm{X}$, deixadas em solução de PBS $1 x$ /formol puro por 5 minutos e passadas novamente duas vezes no tampão PBS IX.

Após essas etapas, as lâminas foram passadas em três concentrações de etanóis gelados $(70 \%, 85 \%$ e $100 \%)$ por 1 minuto cada. Na fase de pré-desnaturação, as lâminas foram deixadas em solução de 2 xSSC/formamida $70 \%$ por 2 minutos a $72^{\circ} \mathrm{C}$ e novamente passadas por $1 \mathrm{mi}-$ nuto em cada concentração de etanol gelado $(70 \%, 85 \%$ e $100 \%$ ). Após a secagem das lâminas, foram colocados $10 \mu \mathrm{l}$ de sonda na região selecionada e esta foi coberta com uma lamínula. Para hibridação, as lâminas foram colocadas na chapa aquecedora a $73^{\circ} \mathrm{C}$ por 10 minutos e logo depois foram armazenadas em caixas úmidas e escuras por aproximadamente 20 horas a $65^{\circ} \mathrm{C}$.

Para a lavagem foi necessário retirar as lamínulas e deixar as lâminas na solução de 2xSSC/0,05\% tween 20 a $37^{\circ} \mathrm{C}$ por 5 minutos, na solução $0,4 x S S X / 0,15 \%$ tween 20 a $73^{\circ} \mathrm{C}$ por 2 minutos, passá-las em seguida em 2 xSSC $/ 0,05 \%$ tween em temperatura ambiente e, posteriormente, em água milique. Após a secagem das lâminas, foram colocados $10 \mu \mathrm{l}$ de DAPI e as regiões selecionadas foram cobertas com lamínulas.

A análise das lâminas foi realizada em microscópio de fluorescência BX51-BF-II/BX2 da Olympus ${ }^{\circledR}$ com filtros de fluorescência adequados e as imagens foram capturadas utilizando o software FISHView da Applied Spectral Imaging ${ }^{\circledR}$. Buscou-se analisar 1.000 núcleos interfásicos de linfócitos e mucosa oral de cada um dos homens saudáveis e dos pacientes com DDS.

Os dados foram analisados por meio do programa computacional SAS System for Windows (Statistical Analysis System), versão 9.2. SAS Institute Inc, 20022008, Cary, NC, USA. O teste de normalidade Shapiro-Wilk foi utilizado para avaliar a distribuição dos dados, já a comparação das medidas numéricas entre indivíduos normais e pacientes com DDS foi feito por meio do teste de Mann-Whitney e a comparação dos resultados obtidos na análise dos dois tipos de células foi feita por meio do teste de Wilcoxon para amostras relacionadas. O nível de significância adotado para os testes estatísticos foi $5 \%$.
O projeto foi aprovado pelo Comitê de Ética em Pesquisa da Faculdade de Ciências Médicas da Unicamp (Parecer 408/2011) e os sujeitos da pesquisa ou seus responsáveis assinaram o termo de consentimento livre e esclarecido.

\section{RESULTADOS}

\section{Validação}

Os achados referentes à análise de núcleos interfásicos de linfócitos e mucosa oral dos homens saudáveis encontram-se na tabela 2. Em ambos os tecidos, o teste de Shapiro-Wilk revelou que essa variável tinha distribuição normal ( $\mathrm{p}=0,105 \mathrm{e} \mathrm{p}=0,082$, respectivamente). A proporção de núcleos contendo apenas o sinal do cromossomo X variou de zero a 14:1.000 em linfócitos de sangue periférico (média: 4,6; desvio-padrão: 3,7 ) e de zero a 13:1.000 em células de mucosa oral (média: 5,2; desvio-padrão: 4,0). Assim, o limite superior da proporção de núcleos contendo apenas o sinal do cromossomo $\mathrm{X}$ para aventar a suspeita de mosaicismo em nosso labo-

Tabela 2. Análise de núcleos interfásicos de linfócitos de sangue periférico e mucosa oral de homens saudáveis por meio de FISH com sondas específicas para os cromossomos $\mathrm{X}$ e $\mathrm{Y}$

\begin{tabular}{|c|c|c|c|c|c|c|}
\hline & & Linfócitos & & & Aucosa ora & \\
\hline & $\begin{array}{c}1 \text { sinal } \\
\text { de } X\end{array}$ & $\begin{array}{c}1 \text { sinal } \\
\text { de } X \text { e } 1 \\
\text { de } Y\end{array}$ & Total & $\begin{array}{c}1 \text { sinal } \\
\text { de } X\end{array}$ & $\begin{array}{c}1 \text { sinal } \\
\text { de } X \text { e } 1 \\
\text { de } Y\end{array}$ & Total \\
\hline 1 & 10 & 990 & 1.000 & 14 & 986 & 1.000 \\
\hline 2 & 3 & 997 & 1.000 & 3 & 997 & 1.000 \\
\hline 3 & 7 & 993 & 1.000 & 6 & 994 & 1.000 \\
\hline 4 & 1 & 999 & 1.000 & 10 & 990 & 1.000 \\
\hline 5 & 3 & 997 & 1.000 & 5 & 995 & 1.000 \\
\hline 6 & 2 & 998 & 1.000 & 4 & 996 & 1.000 \\
\hline 7 & 6 & 994 & 1.000 & 0 & 1000 & 1.000 \\
\hline 8 & 10 & 990 & 1.000 & 8 & 992 & 1.000 \\
\hline 9 & 4 & 996 & 1.000 & 3 & 997 & 1.000 \\
\hline 10 & 5 & 995 & 1.000 & 2 & 998 & 1.000 \\
\hline 11 & 0 & 1000 & 1.000 & 8 & 992 & 1.000 \\
\hline 12 & 0 & 1000 & 1.000 & 2 & 998 & 1.000 \\
\hline 13 & 1 & 999 & 1.000 & 0 & 1000 & 1.000 \\
\hline 14 & 3 & 997 & 1.000 & 3 & 997 & 1.000 \\
\hline 15 & 14 & 986 & 1.000 & 7 & 993 & 1.000 \\
\hline 16 & 7 & 993 & 1.000 & 13 & 987 & 1.000 \\
\hline 17 & 5 & 995 & 1.000 & 4 & 996 & 1.000 \\
\hline 18 & 3 & 997 & 1.000 & 4 & 996 & 1.000 \\
\hline 19 & 4 & 996 & 1.000 & 2 & 998 & 1.000 \\
\hline
\end{tabular}


ratório é de 12:1.000 em linfócitos e 13:1.000 em células de mucosa oral (média mais dois desvios-padrão). Considerando média mais três desvios-padrão, esse limite é de 16:1.000 e 17:1.000, respectivamente.

A comparação da frequência de núcleos contendo apenas o sinal do cromossomo $\mathrm{X}$ nos linfócitos e células de mucosa oral desses indivíduos não mostrou diferença significativa $(\mathrm{p}=0,6855)$.

\section{Aplicação a pacientes com DDS}

Os achados referentes à análise de núcleos interfásicos de linfócitos e mucosa oral dos pacientes com DDS encontram-se na tabela 3. Apenas no caso da paciente 4A não foi possível contar os 1.000 núcleos previstos por haver material insuficiente. Foram detectados núcleos contendo apenas o sinal do cromossomo $\mathrm{X}$ tanto nos quatro casos em que o mosaicismo já havia sido observado no cariótipo convencional quanto no caso 2A, cujo cariótipo era 46,XY em 50 células. Nesse caso, a confirmação da presença da linhagem $45, \mathrm{X}$ foi feita posteriormente por meio do estudo de 144 metáfases também por FISH, sendo encontradas 15 células 45 , X (10,4\%); este caso foi recentemente publicado (18).

A proporção de núcleos contendo apenas o sinal do cromossomo X variou de 76:1.000 a 430:1.000 em linfócitos de sangue periférico (média: 267,4; desvio-padrão: 130,7) e de 234 a 430:1.000 em células de mucosa oral (média: 348,8; desvio-padrão: 76,1). A frequência de núcleos contendo apenas o sinal do cromossomo X diferiu significativamente da observada em indivíduos saudáveis tanto em linfócitos $(\mathrm{p}=0,0008)$ quanto em células de mucosa oral $(\mathrm{p}=0,0008)$.

No caso $5 \mathrm{~A}$ houve ainda outros achados além da presença somente do sinal do $\mathrm{X}$ ou de um sinal de $\mathrm{X}$ e um de $\mathrm{Y}$, indicando a existência de outras linhagens celulares além daquelas detectadas no cariótipo convencional (45,X e 46,XY,del(Y)(q12)). A comparação da frequência de núcleos contendo apenas o sinal do cromossomo X nos linfócitos e células de mucosa oral desses pacientes não mostrou diferença significativa $(\mathrm{p}=0,3750)$.

\section{DISCUSSÃO}

Nas condições técnicas de nosso laboratório, o achado de núcleos interfásicos contendo, na FISH, apenas o sinal do X em número superior a 12:1.000 em linfócitos ou a 13:1.000 em mucosa oral é indicativo da presença de mosaicismo com linhagem $45, \mathrm{X}$ em pacientes $46, \mathrm{XY}$ com DDS ou esterilidade. Além disso, o fato de não haver diferença entre a análise de linfócitos e mucosa oral permite que essa análise seja feita diretamente em células de mucosa.

É importante ressaltar que, embora esse achado teoricamente também possa se aplicar à pesquisa de mosaicismo críptico com linhagem 45,X em pacientes 46,XX com suspeita clínica de ST, novos estudos seriam necessários para comprovar essa hipótese.

O uso da FISH em mucosa oral é uma alternativa não invasiva para investigação de mosaicismo cromossômico, especialmente em casos de complementação diagnóstica em que já não são disponíveis linfócitos cultivados em número suficiente. Considerando o custo ainda elevado dos kits comerciais de FISH, o uso da mucosa em lugar de linfócitos reduz os custos com insumos ao tornar desnecessária nova cultura de células. Além disso, permite a análise de tecido da mesma origem embrionária dos fibroblastos sem exigir biópsia de pele nem cultura de longa duração e utilizando a mesma infraestrutura laboratorial.

Tabela 3. Análise de núcleos interfásicos de linfócitos de sangue periférico e mucosa oral de pacientes com DDS por meio de FISH com sondas específicas para os cromossomos $\mathrm{X}$ e $\mathrm{Y}$

\begin{tabular}{|c|c|c|c|c|c|c|c|c|}
\hline \multirow[b]{2}{*}{ Caso } & \multicolumn{4}{|c|}{ Linfócitos } & \multicolumn{4}{|c|}{ Mucosa oral } \\
\hline & 1 sinal de $X$ & $\begin{array}{c}1 \text { sinal de } X e \\
1 \text { de } Y\end{array}$ & Outros* & Total & 1 sinal de $X$ & $\begin{array}{c}1 \text { sinal de } X e \\
1 \text { de } Y\end{array}$ & Outros* & Total \\
\hline $1 \mathrm{~A}$ & 76 & 924 & 0 & 1.000 & 355 & 645 & 0 & 1.000 \\
\hline $2 \mathrm{~A}$ & 233 & 767 & 0 & 1.000 & 234 & 766 & 0 & 1.000 \\
\hline $3 A$ & 430 & 570 & 0 & 1.000 & 430 & 570 & 0 & 1.000 \\
\hline $4 \mathrm{~A}$ & 331 & 669 & 0 & 1.000 & 324 & 535 & 0 & $859^{\#}$ \\
\hline $5 \mathrm{~A}$ & 267 & 557 & 176 & 1.000 & 401 & 519 & 80 & 1.000 \\
\hline
\end{tabular}

* Núcleos apresentando 1 sinal de $X$ e 2 sinais de $Y$ muito próximos; núcleos com 1 sinal de $X$ e 2 sinais separados de $Y$; e núcleos com 1 sinal de $X$ e 4 sinais de $Y$.\# Material insuficiente para completar a análise de 1.000 núcleos. 
A FISH permite ainda melhor aproveitamento de recursos humanos ao reduzir acentuadamente o tempo de análise para pesquisa de mosaicismo. Em uma preparação cromossômica rica em linfócitos, a análise de 100 células demanda cerca de 4 horas, ao passo que a contagem de 1.000 células de mucosa pode ser realizada em cerca de 3 horas. Por outro lado, a principal desvantagem da FISH em mucosa é a necessidade de processamento do material logo após a coleta, em vista da possível contaminação por bactérias normalmente presentes na cavidade oral.

O cariótipo é o ponto de partida da investigação de pacientes com ambiguidade genital. $\mathrm{O}$ achado de anomalias numéricas ou estruturais de cromossomos sexuais ou quimerismo $(46, \mathrm{XX} / 46, \mathrm{XY})$ está associado a diferentes quadros de distúrbios da diferenciação gonadal (DDG), incluindo DGM e hermafroditismo. O cariótipo 46,XX também pode estar associado a um DDG, mas nesses casos a hiperplasia adrenal congênita é o diagnóstico mais frequente. Por sua vez, o cariótipo 46,XY também pode se associar a um DDG, mas mais frequentemente está presente em pacientes com anomalias na produção ou ação de hormônios testiculares (19).

A partir do resultado do cariótipo, exames hormonais, histopatológicos, moleculares e de imagem são utilizados para prosseguir na investigação e chegar ao diagnóstico etiológico (20). Assim, quando é solicitado o estudo cromossômico são poucos os elementos para estabelecer uma hipótese diagnóstica (somente história clínica e exame físico, na maioria das vezes) e não é possível prever se haverá necessidade de pesquisar mosaicismo por FISH.

Em homens estéreis com genitais masculinos típicos, o exame de cariótipo busca detectar não só a síndrome de Klinefelter (47,XXY) mas também anomalias estruturais do cromossomo Y e anomalias autossômicas aparentemente equilibradas. Revisões recentes sobre o assunto não chegam a mencionar o cariótipo $45, \mathrm{X} / 46, \mathrm{XY}$ como um dos possíveis achados (21), embora este também possa ser encontrado. Assim, ao ser solicitado o exame de cariótipo raramente essa é uma hipótese que já esteja sendo considerada, e também raramente está prevista a necessidade de pesquisar mosaicismo por FISH.

Portanto, não raro a necessidade de buscar uma linhagem 45 , $\mathrm{X}$ em um paciente com DDS ou esterilidade e cariótipo 46,XY surge quando não é mais possível utilizar, para FISH, material da cultura de linfócitos a partir da qual foi analisado o cariótipo. Nesses casos, a análise de núcleos de mucosa oral poderia ser usada.

Considerando que o uso de FISH de mucosa oral ainda não é rotineiro, neste momento não existem pa- râmetros na literatura para estabelecer o intervalo de variação do número de células aparentemente anômalas em controles. Assim, nos casos em que esse exame indicar mosaicismo seria importante a confirmação do diagnóstico em metáfases de linfócitos.

De todo modo, nos casos em que a análise de mucosa por FISH indicar a presença de linhagem $45, \mathrm{X}$, já estaria justificada a pesquisa de anomalias congênitas associadas à presença dessa linhagem, bem como iniciar o seguimento como preconizado para a ST, particularmente quando o número de núcleos anômalos for superior a 17:1.000. Perante valores entre 13 e 17:1.000, a conduta deve ser avaliada caso a caso, considerando o quadro clínico como um todo, incluindo dados antropométricos, exame dismorfológico e características genitais e de função gonadal.

A ampla variação observada nos controles (de zero a 14 células sem o sinal do $\mathrm{Y}$ ) pode se dever a questões técnicas. $\mathrm{O}$ sinal da sonda pode não ser visualizado, por exemplo, por sobreposição de células ou dobramento dos núcleos. No único estudo semelhante encontrado na literatura, foram analisados 200 núcleos de mucosa oral de 15 homens e 15 mulheres saudáveis e nove pacientes com diversos quadros clínicos (22). Os autores determinaram um limite superior de $1 \%$ de núcleos anômalos nos indivíduos do sexo masculino e consideraram a FISH em mucosa oral um exame sensível para diagnóstico de mosaicismo de cromossomos sexuais.

Ao aplicar a técnica de FISH no caso 2A, com diagnóstico prévio de disgenesia gonadal parcial 46,XY, foi possível chegar ao diagnóstico correto de DGM (18) - como já foi visto, em ambos há disgenesia testicular parcial e ambiguidade genital. Com isso, a criança realizou todas as investigações adicionais e passou a receber GH, uma vez que já se comprovou seu benefício na melhora da altura final também na DGM (23).

Nos cinco pacientes com DDS, o número de células com apenas o sinal do $\mathrm{X}$ nos dois tecidos analisados foi muito superior ao limite calculado a partir do estudo dos jovens saudáveis. Esse fato foi observado até mesmo no paciente 2-A, que tinha cariótipo de rotina normal em 50 células. Esse caso pode ser considerado modelo de situação em que o FISH, mesmo que realizado apenas em células de mucosa oral, poderia ser utilizado como exame de rotina para a elucidação do diagnóstico etiológico em casos de DDS 46,XY. Nesses cinco pacientes também não houve diferença significativa entre os achados nos dois tecidos, confirmando que o FISH somente de mucosa oral poderia ser utilizado na rotina. 
No caso 5A, no qual já eram conhecidas duas linhagens pelo cariótipo convencional, uma monossômica e outra com deleção do braço longo do cromossomo $\mathrm{Y}$, achados adicionais em proporção significativa dos núcleos nos dois tecidos indicaram a existência de linhagens adicionais.

Os resultados deste trabalho indicam, portanto, que a pesquisa de mosaicismo com linhagem $45, \mathrm{X}$ em pacientes com DDS ou esterilidade e cariótipo 46,XY pode ser feita por meio de FISH em células de mucosa oral. Isso torna desnecessária a realização de nova cultura de linfócitos (embora esta deva ser realizada buscando confirmar os casos positivos) e pode também substituir a cultura de fibroblastos por biópsia de pele como meio de avaliar a constituição cromossômica em tecido de origem embrionária diversa. Seu emprego tem, portanto, vantagens evidentes em termos de custo e rapidez, além de não ser invasivo.

Agradecimentos: os autores agradecem ao Laboratório de Citogenética Humana e Citogenômica do Departamento de Genética Médica da Faculdade de Ciências Médicas da Universidade Estadual de Campinas (FCM/Unicamp). Este trabalho foi financiado pela Fundação de Amparo à Pesquisa do Estado de São Paulo (Fapesp) (2011/50189-7).

Declaração: os autores declaram não haver conflitos de interesse neste estudo.

\section{REFERÊNCIAS}

1. Nussbaum RL, Mclnnes RR, Willard HF. Princípios de citogenética clínica. In:Thompson \&Thompson Genética Médica. $7^{a}$ ed. Rio de Janeiro: Elsevier; 2008. p. 61-88.

2. Cheung SW, Shaw CA, Scott DA, Patel A, Sahoo T, Bacino CA, et al. Microarray-based $\mathrm{CGH}$ detects chromosomal mosaicism not revealed by conventional cytogenetics. Am J Med Genet A. 2007;143A(15):1679-86.

3. Nussbaum RL, Mclnnes RR, Willard HF. Distúrbios dos autossomos e dos cromossomos sexuais. In: Thompson \& Thompson Genética Médica. $7^{\mathrm{a}}$ ed. Rio de Janeiro: Elsevier; 2008. p. 89-116.

4. Wiktor AE, van Dyke DL. Detection of low level sex chromosome mosaicism in Ullrich-Turner syndrome patients. Am J Med Genet A. 2005;138A:259-61.

5. Bondy CA; Turner Syndrome Study Group. Care of girls and women with Turner syndrome: a guideline of the Turner Syndrome Study Group. J Clin Endocrinol Metab. 2007;92:10-25.

6. Chang HJ, Clark RD, Bachman H. The phenotype of $45, X / 46, X Y$ mosaicism: an analysis of 92 prenatally diagnosed cases. Am J Hum Genet. 1990;46:156-67.
7. Telvi L, Lebbar A, Del Pino O, Barbet JP, Chaussain JL. 45,X/46,XY mosaicism: report of 27 cases. Pediatrics. 1999;104:304-8.

8. Reddy KS, Sulcova V. Pathogenetics of $45, X / 46, X Y$ gonadal mosaicism. Cytogenet Cell Genet. 1998;82:52-7.

9. Guttenbach M, Koschorz B, Bernthaler U, GrimmT, Schmid M. Sex chromosome loss and aging: in situ hybridization studies on human interphase nuclei. Am J Hum Genet. 1995;57:1143-50.

10. Hunter S, Gramlich T, Abbott K, Varma V. Y chromosome loss in esophageal carcinoma: an in situ hybridization study. Genes Chromosomes Cancer. 1993;8:172-7.

11. Tagliarini EB, Assumpção JG, Scolfaro MR, Mello MP, Maciel-Guerra AT, Guerra Júnior G, et al. Mutations in SRY and WT1 genes required for gonadal development are not responsible for XY partial gonadal dysgenesis. Br J Med Biol Res. 2005;38:17-25.

12. Lin $L$, Achermann JC. Steroidogenic factor-1 (SF-1, Ad4BP, NR5A1) and disorders of testis development. Sex Dev. 2008;2:200-9.

13. Kearney L. Molecular cytogenetics. Best Pract Res Clin Haematol. 2001;14:645-68.

14. Barros BA, Moraes SG, Coeli FB, Assumpção JG, De Mello MP, Maciel-Guerra AT, et al. OCT4 immunohistochemistry may be necessary to identify the real risk of gonadal tumors in patients with Turner syndrome and $Y$ chromosome sequences. Hum Reprod. 2011;26:3450-5.

15. Hook EB. Exclusion of chromosome mosaicism: tables of 90 percent, 95 percent and 99 percent confidence limits and comments on use. Am J Hum Genet. 1977;29:94-7.

16. Nazarenko SA, Timoshevsky VA, Sukhanova NN. High frequency of tissue-specific mosaicism in Turner syndrome patients. Clin Genet. 1999;56:59-65.

17. Romczak EZ, Punnett HH. An improved method for chromosome enumeration using fluorescent in situ hybridization of buccal epithelium. J Ass Genet Tech. 1998;24:6-7.

18. Maciel-Guerra AT, De Paulo J, Santos AP, Guaragna-Filho G, Andrade JGR, Siviero-Miachon AA, et al. The use of fluorescence in situ hybridization in the diagnosis of hidden mosaicism: a propos of three cases of sex chromosome anomalies. Arq Bras Endocrinol Metab. 2012;56(8):545-51.

19. Lee PA, Houk CP, Ahmed SF, Hughes IA; International Consensus Conference on Intersex organized by the Lawson Wilkins Pediatric Endocrine Society and the European Society for Pediatric Endocrinology. Consensus statement on management of intersex disorders. International Consensus Conference on Intersex. Pediatrics. 2006;118:488-500.

20. Guerra-Junior G, Maciel-Guerra AT. The role of the pediatrician in the management of children with genital ambiguity. J Pediatr (Rio J). 2007;83:S184-91.

21. McLachlan RI, O'Bryan MK. Clinical Review\#: state of the art for genetic testing of infertile men. J Clin Endocrinol Metab. 2010;95:1013-24.

22. Schad CR, Kuffel DG, Wyatt WA, Zinsmeister AR, Jenkins RB, Dewald GW, et al. Application of fluorescent in situ hybridization with $\mathrm{X}$ and $\mathrm{Y}$ chromosome specific probes to buccal smear analysis. Am J Med Genet. 1996;66:187-92.

23. Johansen ML, Hagen CP, Rajpert-De Meyts E, Kjærgaard S, Petersen $B L$, Skakkebæk NE, et al. 45,X/46,XY mosaicism: phenotypic characteristics, growth, and reproductive function - a retrospective longitudinal study. J Clin Endocrinol Metab. 2012;97:E1540-9. 